\title{
Model-Based Quantification of Load Shift Potentials and Optimized Charging of Electric Vehicles
}

\author{
Tobias Hahn", Martin Schönfelder ${ }^{2}$, Patrick Jochem², Vincent Heuveline ${ }^{3}$, Wolf Fichtner ${ }^{2}$ \\ ${ }^{1}$ Institute of Process Engineering in Life Sciences, Karlsruhe Institute of Technology (KIT), Karlsruhe, Germany; ${ }^{2}$ Chair of Energy \\ Economics, Karlsruhe Institute of Technology (KIT), Karlsruhe, Germany; ${ }^{3}$ University of Heidelberg, Interdisciplinary Center for \\ Scientific Computing, Heidelberg, Germany. \\ Email: tobias.hahn@kit.edu, martin.schoenfelder@kit.edu, patrick.jochem@kit.edu, vincent.heuveline@iwr.uni-heidelberg.de, \\ wolf.fichtner@kit.edu
}

Received June $4^{\text {th }}, 2013$; revised July $4^{\text {th }}, 2013$; accepted July $11^{\text {th }}, 2013$

Copyright (C 2013 Tobias Hahn et al. This is an open access article distributed under the Creative Commons Attribution License, which permits unrestricted use, distribution, and reproduction in any medium, provided the original work is properly cited.

\begin{abstract}
Managing the charging process of a large number of electric vehicles to decrease the pressure on the local electricity grid is of high interest to the utilities. Using efficient mathematical optimization techniques, the charging behavior of electric vehicles shall be optimally controlled taking into account network, vehicle, and customer requirements. We developed an efficient algorithm for calculating load shift potentials defined as the range of all charging curves meeting the customer's requirements and respecting all individual charging and discharging constraints over time. In addition, we formulated a mixed integer linear program (MIP) applying semi-continuous variables to find cost-optimal load curves for every vehicle participating in a load shift. This problem can be solved by e.g. branch-and-bound algorithms. Results of two scenarios of Germany in 2015 and 2030 based on mobility studies show that the load shifting potential of $\mathrm{EV}$ is significant and contribute to a necessary relaxation of the future grid. The maximum charging and discharging power and the average battery capacity are crucial to the overall load shift potential.
\end{abstract}

Keywords: Electric Mobility; Load Shifting; Charging Management; Optimization

\section{Introduction}

Tomorrow's energy systems will exhibit a tremendous complexity reflected mainly by their dynamic behavior and heterogeneity. This will apply to Germany in particular because of the constantly increasing share of power generation from renewable energy sources (RES) as well as the ongoing decentralization of the power system. The regionally strongly differing potentials of RES will lead to an increasing spatial imbalance of electricity generation and demand. Moreover, their fluctuating character will require various measures, such as more flexible power plants, a growing capacity of efficient energy storage systems, and the application of Demand Side Management (DSM) methods.

At the same time, ongoing and extensive research and development activities regarding electric vehicles (EV) will facilitate the latters' market penetration. A significant share of EV in the passenger car fleet will lead to an increasing demand for electric energy especially during peak hours, if no controlled charging strategy is applied [1]. This could jeopardize the local power grid [2].
However, due to the normally long parking times of vehicles used for individual mobility purposes, the degrees of freedom in charging EV are comparably high [3]. Therefore, controlled (e.g. delayed) charging of EV as one DSM approach can help to avoid load peaks. Moreover, controlled charging strategies could support the integration of fluctuating electricity supply based on RES [4,5]. Even more services can be provided, if the possibility of feeding electricity back into the grid is considered (e.g. auxiliary services [6]).

To determine the technical potential of load shifting by $\mathrm{EV}$, the complex interplay of millions of consumers and stationary and mobile energy producers has to be evaluated with many specific restrictions and different levels of aggregation and control points. This task is highly complex in view of the heterogeneity and variety of individual objects, which additionally differ in their temporal availability.

Using efficient mathematical optimization techniques, the charging behavior of electric vehicles shall be optimally controlled taking into account network, vehicle, 
and customer requirements. For individual vehicles connected to a charging station and given electricity price levels, a cost minimizing charge function is determined with the customer requirements being taken into consideration. The local load shifting potential is defined as the range of alternative charging functions that meet the customer requirements. However, not all of these charging functions may be economically optimal for the customer. The electricity supplier therefore needs to pay for the customer's permission to choose a different load curve that better fulfills the global conditions (e.g. planed generating capacity, network utilization, etc.). In this way, system efficiency can be increased considerably by appropriate Demand Side Management.

This paper presents a model to compute the range of actual load shift potentials under the constraints of individual preferences, technical restrictions, and the electricity demand of EV. The model will be used as a basis for further investigations, e.g. to optimize the network utilization under macro-economic and energy-economic criteria by adjusting the charging functions of each involved EV with respect to individual technical restrictions. Recently, numerous works were published in the research field addressed by this paper. Especially the optimization of EV charging subject to network constraints is addressed with several models seeking to determine grid-compatible charging strategies [7-10] or simulating the grid effects of different charging strategies [1,11,12]. Other works focus on methods to influence consumer behavior to achieve grid-optimal EV charging [13,14]. Some Multi-Agent Systems (MAS) have also been proposed [15-17] to achieve a decentralized optimal behavior. In contrast to the works mentioned, our focus is on the online determination of load shift potentials for the next hours. This is the relevant information for the local distribution system operator (DSO). Furthermore, our model allows for the use of actual load shifts within the simulated potentials (e.g. by a central instance). The software updates the aggregated potentials of the future without neglecting the underlying constraints. This is achieved by constantly re-optimizing the distributed charging strategies subject to updated constraints.

The paper is divided into seven sections. Section 2 introduces the mathematical model to calculate the aggregated load shift potential. Besides the formal definition of load curves, the mathematical description of the individual as well as of the aggregated load shift potential is given. In the third section the optimization problem for the generation of optimal load curves is presented. Furthermore, the optimization problem is adapted to determine the upper and lower limits of the charging functions used to compute the load shift potential of all vehicles. In Section 4, DSM operation and processing of load shifts are described. Section 5 covers the application of the model to two realistic scenarios while Section 6 gives an outlook before coming to the conclusions in Section 7.

\section{Mathematical Model}

This section describes all quantities and mathematical definitions involved in the optimization problem to be solved in Section 3. The load curve of a vehicle can be any function in time, which fulfills the customer requirements and follows the technical restrictions. The main customer requirement is a full battery at the end. The technical restrictions include upper and lower limits for the charge and feed power. The upper limits are usually imposed by the battery or charging station, while the lower limits are due to a certain threshold which ensures a flow of energy.

Furthermore, the available amount of energy shall not drop below the minimum reserve and the battery's capacity must not be exceeded. For the grid, the quantities of interest are the amounts of energy required and available rather than the individual states of charge or reserve levels. The constraints are formulated with the help of these quantities in the sections below.

\subsection{Load Curve}

For a vehicle $V$ that is to be charged with a certain amount of energy $E_{V}^{+} \geq 0$ in a time interval $T_{V}=$ $\left[t_{b}, t_{e}\right]$, the load curve $L_{V}(t)$ to reach the desired amount of energy during charging time is required, i.e.

$$
\int_{T_{V}} L_{V}(t) \mathrm{d} t=E_{V}^{+},
$$

where we assume, that this is technically feasible. In case the charging time is not sufficient to fill the battery, the desired amount of energy shall be set to the amount that can be reached.

The maximum capacity constraint can be expressed by staying below the required amount of energy at any point of the charging process,

$$
\int_{t_{b}}^{t} L_{V}(t) \mathrm{d} t \leq E_{V}^{+} \quad \forall t<t_{e}
$$

The same applies to the amount of available regenerative energy $E_{V}^{-} \geq 0$,

$$
-\int_{t_{b}}^{t} L_{V}(t) \mathrm{d} t \leq E_{V}^{-} \quad \forall t<t_{e} .
$$

The technical restrictions include upper and lower limits for the charge and feed power.

$$
\begin{array}{ll}
0<P_{V, \text { min }}^{+}(t) \leq L(t) \leq P_{V, \text { max }}^{+}(t) & \forall t: L(t)>0, \\
0>P_{V, \text { min }}^{-}(t) \geq L(t) \geq P_{V, \text { max }}^{-}(t) \quad \forall t: L(t)<0 .
\end{array}
$$

Figure 1 shows an example of a load curve within the admissible ranges. The limits as well as the load curve may be discontinuous. In this case, the load curve is piecewise constant. 


\subsection{Individual Load Shift Potential}

Usually, there is a large number of load curves $L_{V}^{(i)}$ which satisfy the conditions in 2.1. The range of all these load curves then defines the load shift potential $S(t)$ :

$$
S(t)=\bigcup_{i} L_{V}^{(i)}(t) .
$$

This means that for every point in $S$, we can find at least one load curve passing this point and thus allowing for shifting this point in time. As the load shift potential is a subset of the technically admissible range of load curves, $S$ is a union of three disjoint sets:

$$
\begin{aligned}
& S(t) \\
& =:\left[S_{V, \text { max }}^{-}(t), S_{V, \text { min }}^{-}(t)\right] \cup\{0\} \cup\left[S_{V, \text { min }}^{+}(t), S_{V, \text { max }}^{+}(t)\right] .
\end{aligned}
$$

The colored areas in Figure 1 show the subsets. If the EV arrives with a low battery level, it cannot discharge in the beginning, which is depicted by the growing lower area. In order to fulfill constraint (1), the EV cannot discharge in the end, which is why the lower area is shrinking again.

\subsection{Accumulated Load Shift Potential of Vehicles}

For the DSM, it is reasonable to divide the load shift potentials into three sub-potentials for the producible, unavoidable, and compensable load:

- Negative load shift potential (load generation): The area between maximum load generation and the current load curve

$$
R^{-}(t):=\sum_{V}\left(S_{V, \max }^{+}(t)-L_{V}(t)\right),
$$

- Positive load shift potential (load reduction): The area between the current load curve and the minimum load generation

$$
R^{+}(t):=\sum_{V} \max \left\{0, L_{V}(t)-S_{V, \text { min }}^{+}(t)\right\},
$$

- Super-positive load shift potential (power generation):

The area between 0 (or the current load curve, if power is currently generated) and the maximum power generation

$$
R^{*}(t)=\sum_{V}\left(\min \left\{0, L_{V}(t)\right\}-S_{V, \max }^{-}(t)\right) .
$$

Figure 2 shows the load shift potential for a vehicle, which is unable to discharge in the beginning. This is the result of a common requirement: The battery is first charged to a specified minimum with full power in order to be able to reach important places, e.g. a hospital. In the end, the upper level might be reduced due to reservation of power for preconditioning of the vehicle, see Section 4.1.

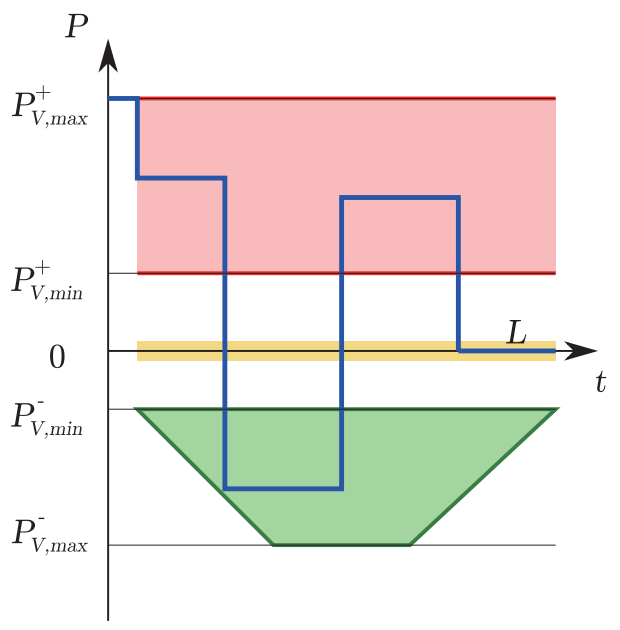

Figure 1. Example load curve and range of values.
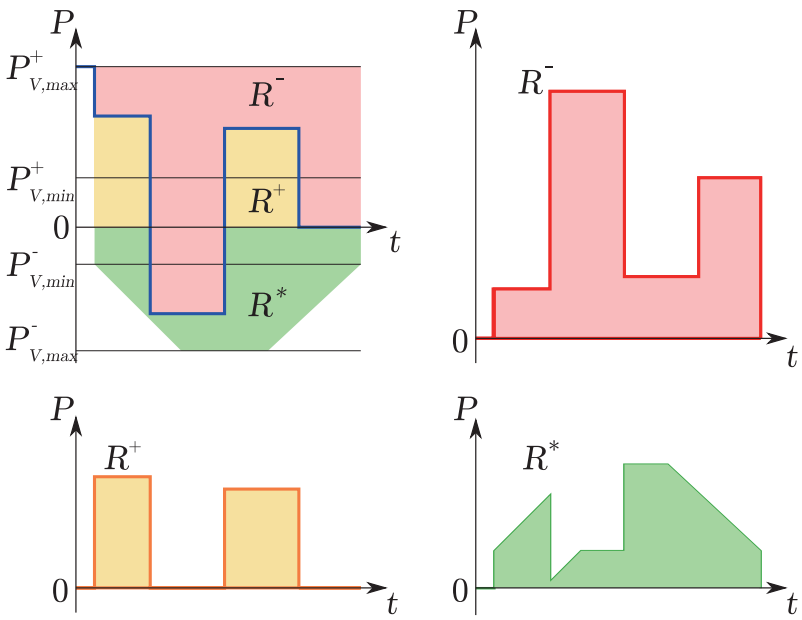

Figure 2. Exemplary load shift potentials for one vehicle.

Dividing the load shift potential into three sub-potentials allows to draw them separately versus the nonmanageable grid load, as shown in Figure 3. The lower edge of $R^{+}$may provide additional information on the unavoidable load when it deviates from the grid load. The impact of vehicle-to-grid functionality can be directly read from $R^{*}$, as only participating vehicles add to it.

\section{Optimization Problem}

DSM starts with offering a time-flexible tariff taking into account all expected fluctuations in electricity generation and demand which is supposed to make the customer schedule highly energy-consuming activities accordingly. Other cost functions are also possible, e.g. based on the $\mathrm{CO}_{2}$ emissions. In reality, however, the reaction to dynamic tariffs depends on the customer's acceptance [18], which is why we assume only reasonably behaving customers. Furthermore, there should be a tariff for feeding energy from the battery into the grid, which should not correspond to the charging tariff in the same interval. 


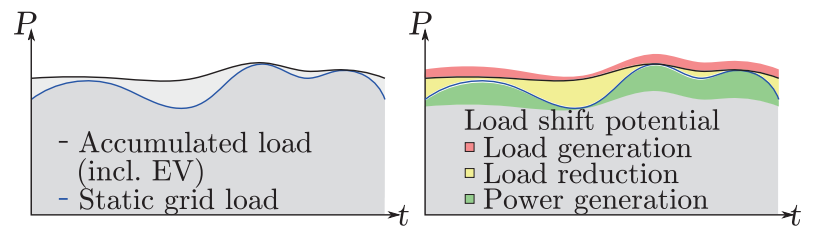

Figure 3. Accumulated load shift potential for all vehicles shown with non-manageable (static) load.

Otherwise, a cost-optimal load curve might be oscillating instead of just staying at zero.

\subsection{Generation of Optimal Load Curves}

Finding a cost-optimal load curve for a complex tariff with case differentiation or a direct algorithm will be difficult in the future when taking into account more complex constraints like battery wear and other physical effects. Even now, the absolute tariffs are not the only decisive factor. Their ratios have to be considered as well. It is thus reasonable to calculate the solution by formulating an optimization problem. Non-linearities are to be avoided in order to apply faster linear programming techniques.

An optimal load curve $L_{V, \text { opt }}$ is defined as an admissible load curve that minimizes the cost for a given tariff/weight function $W(t)$ :

$$
L_{V, \text { opt }}(t)=\min _{L(t)} \int_{T_{V}} L_{V}(t) \cdot W(t) \mathrm{d} t .
$$

As there are two intervals enclosing zero, where charging or discharging is not allowed, the domain becomes non-convex. Without simplification, solution of the optimization problem will require a large expenditure [19]. Hence, we discretize the problem and construct constraints in order to select values from the admissible range only.

We can expect the cost function to be piecewise constant in the future, as all other forms of tariff will most probably over-strain the customer. Without loss of generality, we expect the intervals where the tariff/weight function $W(t)$ is constant to be of constant length (e.g. one hour), such that we can use a vector form $\bar{W}=\left\{w_{i}\right\}$.

As we are only interested in non-oscillating load curves which are constant over the time intervals, we use the same vectorized form to define load curve representations $\bar{L}$.

We now consider different tariffs for charging and feeding

$$
w_{i}\left(l_{V, i}\right)=\left\{\begin{array}{ll}
w_{i}^{+} & \text {for } l_{V, i} \geq 0 \\
w_{i}^{-} & \text {for } l_{V, i}<0
\end{array} .\right.
$$

To avoid case differentiation, $\bar{L}_{V}$ is decomposed into charging and discharging curves, as shown in Figure 4,

$$
\bar{L}_{V}=\bar{Z}_{V}^{+}+\bar{Z}_{V}^{-} \text {. }
$$

The objective is then reformulated to

$$
\min _{\bar{Z}_{V}^{+}, \bar{Z}_{V}^{-}} \bar{W}^{+} \cdot \bar{Z}_{V}^{+}+\bar{W}^{-} \cdot \bar{Z}_{V}^{-} .
$$

It has to be ensured that both curves lie within the allowed ranges and charging and discharging do not happen at the same time. The admissible ranges are

$$
\begin{array}{lll}
\bar{z}_{V, i}^{+}=0 & \vee & z_{V, i}^{+} \in\left[\bar{P}_{V, \text { min }, i}^{+}, \bar{P}_{V, \text { max }, i}^{+}\right], \\
\bar{z}_{V, i}^{-}=0 & \vee & z_{V, i}^{-} \in\left[\bar{P}_{V, \text { max }, i}^{-}, \bar{P}_{V, \text { min }, i}^{-}\right] .
\end{array}
$$

Many modern mixed integer programming (MIP) solvers directly support this kind of semi-continuous variables, such that no additional integers have to be manually introduced to fulfill the either-or condition above.

On the other hand, without adding further variables, the constraint that only one of the functions $\bar{Z}_{V}^{+}$and $\bar{Z}_{V}^{-}$can be non-zero at a time, can only be written in a bilinear form,

$$
\bar{Z}_{V}^{+} \cdot \bar{Z}_{V}^{-} \equiv 0,
$$

which is not allowed in most conventional MIP solvers. Specialized algorithms exist [20], but usually take more computing time. We hence introduce binary indicator functions

$$
\bar{C}_{V}, \bar{F}_{V}=\{0,1\}^{n}
$$

as also shown in Figure 4. The additional constraints

$$
\begin{gathered}
c_{V, i} \cdot \bar{P}_{V, \max , i}^{+} \geq z_{V, i}^{+} \forall i, \\
f_{V, i} \cdot \bar{P}_{V, \max , i}^{-} \leq z_{V, i}^{-} \forall i,
\end{gathered}
$$

ensure the correct setting of $\bar{C}_{V}$ and $\bar{F}_{V}$. For example, $z_{V, i}^{+}>0$ implies $c_{V, i}=1$.

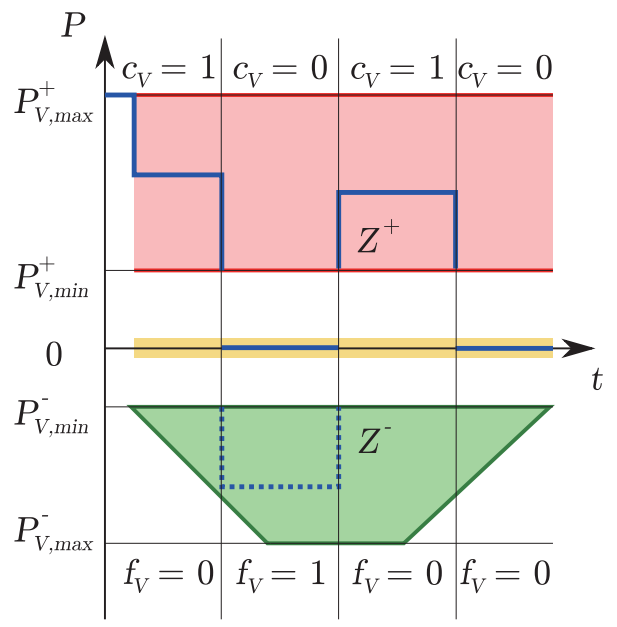

Figure 4. Decomposition into charging and discharging curves and introduction of binary indicators. 
The substitution of $\bar{Z}_{V}^{+} \cdot \bar{Z}_{V}^{-} \equiv 0$ is given by

$$
c_{V, i}+f_{V, i} \leq 1 \forall i \text {. }
$$

Finally, the constraints formulated in (1)-(3) can be rewritten for a charging time of $n$ intervals of length $\Delta t$ :

- Full charging

$$
\Delta t \sum_{i=1}^{n}\left(z_{V, i}^{+}+z_{V, i}^{-}\right)=E_{V}^{+},
$$

- Upper capacity limit

$$
\Delta t \sum_{i=1}^{k}\left(z_{V, i}^{+}+z_{V, i}^{-}\right)<E_{V}^{+} \quad \forall_{k<n}
$$

- Lower capacity limit

$$
-\Delta t \sum_{i=1}^{k}\left(z_{V, i}^{+}+z_{V, i}^{-}\right)<E_{V}^{-} \quad \forall_{k<n} .
$$

This final formulation is completely linear, it consists of the objective (14), constraints (19)-(24), and admissible ranges (15), (16) and (18).

The problem can be solved with established methods like branch-and-bound [21] or branch-and-cut, which extends the former algorithm by cutting planes. The implementation chosen here is lp_solve [22], a free branchand-bound solver supporting semi-continuous variables.

In case semi-continuous variables are not available, two additional constraints are needed:

$$
\begin{gathered}
c_{V, i} \cdot \bar{P}_{V, \text { min }, i}^{+} \leq z_{V, i}^{+} \forall i, \\
f_{V, i} \cdot \bar{P}_{V, \text { min }, i}^{-} \geq z_{V, i}^{-} \forall i .
\end{gathered}
$$

In this way, the problem can be solved with conventional MIP routines as offered by computing environments like Maple. As the integer variables are binary, it is possible to consider all configurations and solve a set of linear problems only. This is no practical method, but punctuates the global optimality of the solution.

From the variety of heuristic methods, simulated annealing would be suited well for mixed integer problems. However, as the computation times with lp_solve are in the range of a few seconds even for large load shifts on a standard dual-core office PC, they were not considered. The degrees of freedom of the optimization problem can also be reduced by joining intervals with equal cost values.

\subsection{Load Shift Potential}

The method described above can also be used to compute the load shift potential by changing the objective function to finding the charge curve reaching a maximum or minimum value at a certain point in time. Four optimization problems would have to be solved for every vehicle and point in time, which would be associated with too high a computational expenditure. As the objective func- tions are simpler in this case, a direct algorithm can be formulated for determining the four boundaries:

$\bar{S}_{V, \text { min }}^{+}$The algorithm has to deliver the requested amount of energy without exceeding the upper and lower limits of the battery capacity. The maximum amount of energy within the charging window is given by $\Delta t \cdot \sum_{j} \bar{P}_{V, \text { max }, j}^{+}$. Subtracting $E_{V}^{+}$results in the amount of energy which is not needed for charging.

The lower power limit of each interval can be found by shifting this area within the charging window, such that we can calculate $\left(\bar{S}_{V, \text { min }}^{+}\right)_{i}$ as the minimum power level necessary by

$$
\begin{aligned}
& \bar{S}_{V, \text { min }, i}^{+} \\
& =\min \left\{\bar{P}_{V, \text { min }, i}^{+} \bar{P}_{V, \text { max }, i}^{+}-\left(\sum_{j} \bar{P}_{V, \text { max }, j}^{+}-\frac{1}{\Delta t} E_{V}^{+}\right)\right\} .
\end{aligned}
$$

$\bar{S}_{V, \text { max }}^{+}$To find the upper limit, the amount of energy not needed for charging has to be shifted more specifically: When discharging at a high state of charge, the amount of chargeable energy increases. This means that charging should start with full power from interval $i$ until the end or until $E_{V}^{+}$is reached.

If we do not have $\bar{S}_{V, \max , i}^{+}=\bar{P}_{V, \max , i}^{+}$yet, we try discharging from interval 1 to $i-1$, such that we can increase the power level at $i$ in a stepwise manner.

$\bar{S}_{V \text {, max }}^{-}$The discharging power limit can be computed similarly: The higher the state of charge, the more energy can be discharged. This means that charging starts with full power, leaving out the interval $i$ and bearing the upper limit $E_{V}^{+}$in mind. When the battery capacity is reached, $\bar{S}_{V, \max , i}^{-}$is lowered in steps to $\bar{P}_{V, \max , i}^{-}$as long as there are intervals left for charging.

$\bar{S}_{V, \min }^{-}$This boundary is equal to $\bar{S}_{V, \text { min }}^{-}$, if $\bar{S}_{V, \max , i}^{-} \leq$ $\bar{P}_{V, \min , i}^{-}$, otherwise it is set to zero.

\section{DSM Operation}

The accumulated load shift potential is highly time-dependent, as vehicles are constantly arriving and departing. At the same time, the set of alternative load curves for each connected EV is constantly reduced when values are fixed over time.

The operation of the DSM, including this update of potentials, is given in Listing 1. In a time step, arrival events have to be processed first (Line 2), then all remaining vehicles are updated with the amount of energy they received during the last interval. Departing vehicles are automatically removed in this step.

At this point, all information items have been updated to compute the load shift potential (Line 3) and process 


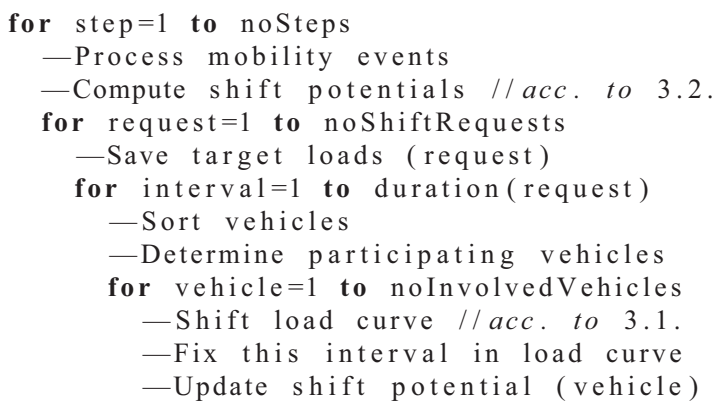

Listing 1. Overview of the DSM cycle.

possible load shift requests (Line 4). Load shift requests are issued by the supplier in reaction to unforeseen deviations from the planned load curve or because of economic considerations. The direct algorithm for computation of potentials is cheap and can be parallelized. Processing of load shift requests can be distributed as well, but requires more communication to exactly achieve the requested shift.

Load shifts over several intervals are processed in steps in temporal order (Line 6). The average goal values are saved, as the cumulative load changes while shifting (Line 5). A request is first compared to the potentials, thereafter the satisfiable part of it is assigned to the participating vehicles. Normally, the vehicles with the longest remaining charging time offer the highest flexibility and load shift potential. Hence, they are sorted in descending order according to their departure times (Line 7).

The partial shifts are performed with a greedy algorithm. For one vehicle after another (Line 9), the maximum possible shift is performed by generating a new curve (Line 10) which attains a minimum or maximum. When processing the next partial load shift, the previously treated intervals are fixed (Line 11), such that the achieved shift cannot be reverted accidentally.

Temporal resolution influences the runtime and accuracy of the simulation. On the one hand, it seems reasonable to choose a small time step for more precise load curves, as vehicles are constantly arriving and departing. On the other hand, a longer interval gives more reliable information on the shift potential. When processing shifts over several intervals, the potential changes after the first step, such that the request might not be fulfilled in the second. In this sense, a load shift is understood to be an averaged shift referred to the medium load value in the interval and longer time steps of 5 to 10 minutes are preferred. Figure 5 shows the load curve and potentials of one vehicle in a demonstrator application.

\subsection{Preconditioning}

Preconditioning demonstrates exemplary the integration of further constraints into the model. Constant energy

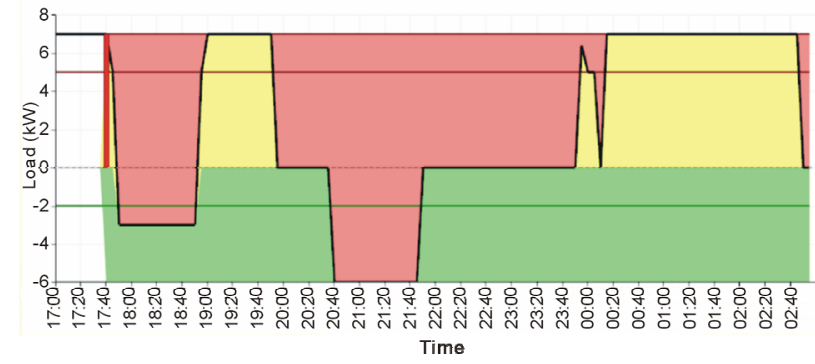

Figure 5. Exemplary vehicle with load curve (black), upper and lower power limits (red/green) and shift potentials (areal). For a sample tariff, discharging between 17:45 and 18:45 as well as between $20: 30$ and $21: 30$ is beneficial and full charging postponed accordingly.

supply during parking and the strictly limited amount of energy while moving make it reasonable to start air conditioning before leaving. Depending on the difference to the preferred temperature, preconditioning is started 10 to 20 minutes before departure. The necessary amount of energy for heating or cooling is calculated from the thermal energy

$$
E=c_{\text {heat }} \cdot m \cdot \Delta T \text {. }
$$

The outside temperature curve is specified in the XML data and the vehicle is assumed to arrive with the goal temperature.

The product of mass and heat capacity is set to a typical value of $100 \mathrm{~kJ} / \mathrm{kg}$ and thermal transfer through the vehicle body to $75 \mathrm{~W} / \mathrm{K}$ [23].

To calculate the amount of energy for preconditioning, we first determine the interior temperature at the beginning of preconditioning via

$$
\begin{aligned}
& T_{\text {in }}(t)=\Delta t \cdot \frac{100 \mathrm{~kJ}}{75 \mathrm{~W}}\left(T_{\text {out }}(t-1)-T_{\text {in }}(t-1)\right) . \\
& T_{\text {in }}(0)=T_{\text {goal }} .
\end{aligned}
$$

We now assume that the target temperature is approached linearly and, hence, compute thermal transfer during preconditioning as

$$
P_{\text {trans }}=75 \frac{W}{K} \cdot \frac{1}{2}\left|T_{\text {goal }}-T_{\text {in }}\right| .
$$

The heating or cooling power is then

$$
P_{a / c}=100 \frac{\mathrm{kJ}}{\mathrm{K}} \cdot\left|T_{\text {goal }}-T_{\text {in }}\right| \cdot \frac{1}{t_{\text {prec }}} .
$$

In order to obtain the final value, which is subtracted from the upper power limit $\bar{P}_{V \text {,max }}^{+}$, we assume a typical coefficient of performance of 1.52 and a ventilation consumption of $500 \mathrm{~W}$ [23].

$$
P_{\text {prec. }}=\frac{P_{a / c}+P_{\text {trans }}}{1.52}+500 \mathrm{~W}
$$




\section{Exemplary Application}

To quantify the potentials, two scenarios are constructed as outlined in Table 1. The results given in the following sections have to be interpreted as an upper limit for the load shift potential, since it is assumed that all vehicles are connected to the grid during parking time.

Scenario 1 (year 2015) is based on the goals of the German Federal Government, which were developed in the so-called National Electric Mobility Platform (NPE) and published in its second report [24]. Scenario 2 (year 2030) is based on a forward projection of these goals, as shown in Figure 6.

The data on the behavior of the vehicles are required from a representative German mobility study which is based on realistic driving profiles for more than 32,000 distinct routes [25]. Due to the limited size of the data set, only some of the EV assumed to exist in total are explicitly represented in the simulation. As a simple scaling of the data set would not have increased the significance, each scenario is limited to 10,000 vehicles and approx. 16,000 distinct routes. This number of vehicles corresponds to the size of the original data set and the assumptions regarding the participation in DSM measures. However, the calculated load shift potentials are scaled up to the total number of EV in each scenario.

Table 1. Basic information on applied scenarios.

\begin{tabular}{ccc}
\hline Parameter & Scenario 1 & Scenario 2 \\
\hline Year & 2015 & 2030 \\
Duration (starting at 12 noon) & 24 hours & 24 hours \\
Total number of EV on the road & 200,000 & $6,000,000$ \\
Vehicles participating in DSM & $50 \%$ & $90 \%$ \\
Maximum power at home & $3.68 \mathrm{~kW}$ & $10 \mathrm{~kW}$ \\
Maximum power at public places & $10 \mathrm{~kW}$ & $22 \mathrm{~kW}$ \\
Number of selected vehicles & 10,000 & 10,000 \\
Approx. number of distinct & 16,000 & 16,000 \\
routes & 20 & 600 \\
Scale-up factor & $60 \%$ & $40 \%$ \\
Share of hybrid EV & $9635 \mathrm{Wh}$ & $18,239 \mathrm{Wh}$ \\
Average battery capacity & $30 \%$ & $50 \%$ \\
\hline Vehicles participating in V2G & & \\
\hline
\end{tabular}

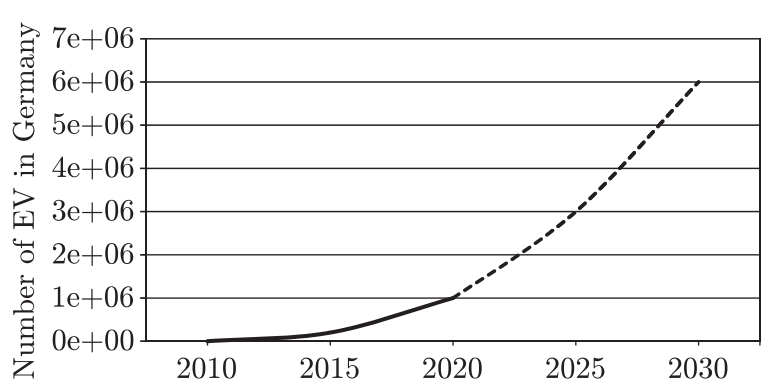

Figure 6. Predicted number of EV in Germany.
The simulation uses a predefined static load curve which is independent of the power demand of the EV. This exogenous load curve correlates with the predefined tariffs shown in Figure 7 and illustrates a realistic load situation. Preconditioning was not considered in these examples to produce more general results without any dependencies on a given whether situation.

\subsection{Scenario 1 (Year 2015)}

The NPE goals [24] include one million EV on German roads in the year 2020. A non-linear extrapolation using the current number of vehicles and the NPE schedule leads to an approximate number of 200,000 vehicles in the year 2015 as shown in Figure 6. In this scenario, the average battery capacity is $9635 \mathrm{Wh}$ because of the comparably high share of hybrid EV which generally have a significantly lower battery capacity than pure EV. Furthermore the share of vehicles which participate in V2G is only $30 \%$ due to an assumed lack of acceptance.

After initialization, the DSM computes the aggregated load shift potential of the currently connected vehicles. Perfect foresight is assumed regarding the departure of connected vehicles, but not for new arrivals. This leads to a realistic representation of the degrees of freedom for the simulated DSM to respond to load shift requests in each individual time step. Running the simulation without any load shift requests leads to a bare visualization of the varying potentials over the corresponding time horizon. Table 2 shows selected results, the notations refer to the definitions given in Section 2.3.

The results show that this setup already leads to significant load shift potentials. Especially the constantly high negative potential is remarkable. However, if the execution of a load shift is requested, the corresponding potentials in future time slots will decrease significantly. Figures 8 and 9 show the aggregated load shift potential over the considered time horizon, without taking the possible realization of load shifts into account. As the load shift potential changes in each time step $t_{i}$ it is in fact two-dimensional. The curves in Figures 8 and 9 show the specific load shift potential available at $t_{i}$ when the point in time $t=t_{i}$ is reached.

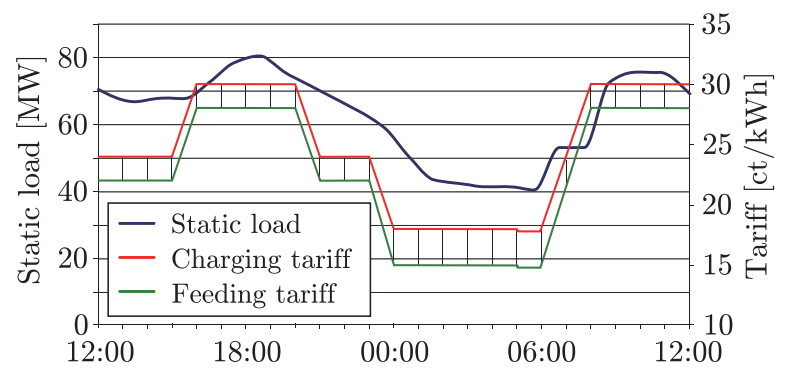

Figure 7. Predefined grid load curve and tariffs for the simulation. 


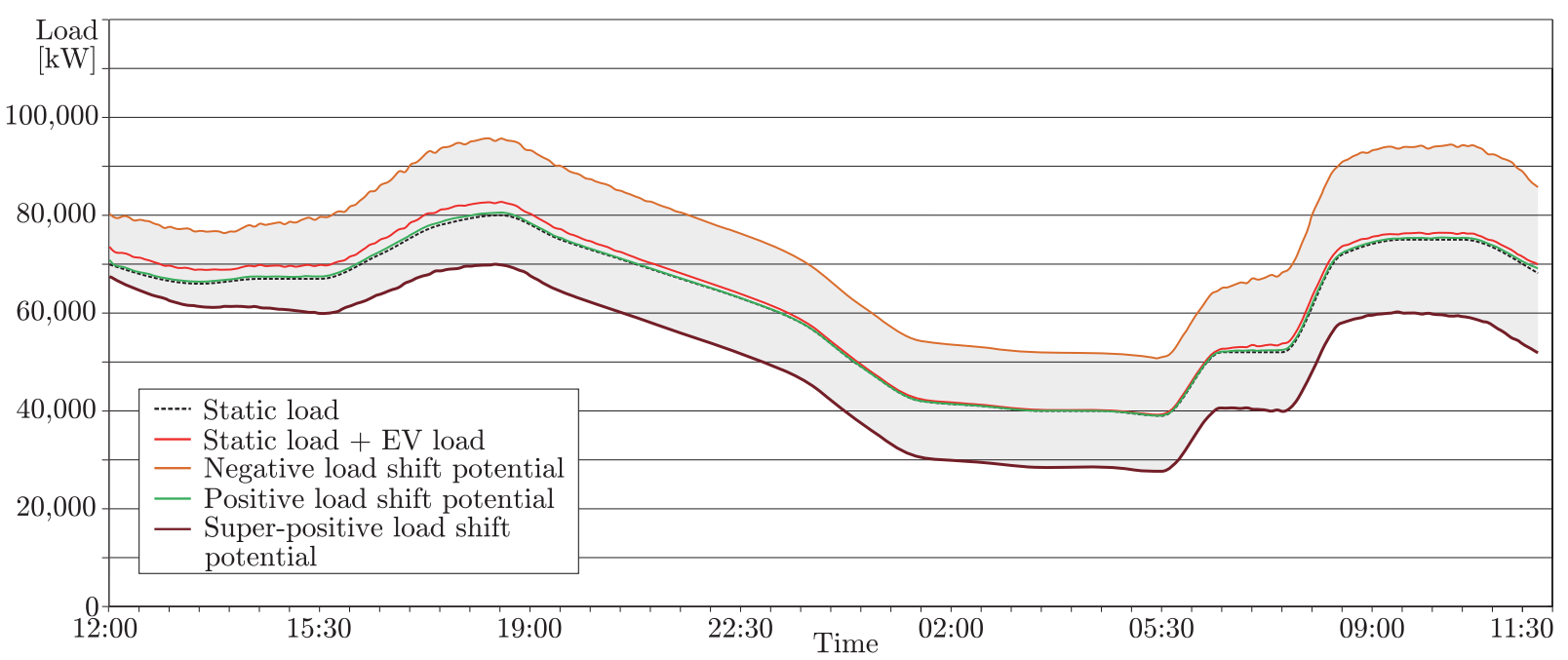

Figure 8. Resulting limits of load shift potentials (10,000 EV) in Scenario 1 (Year 2015).

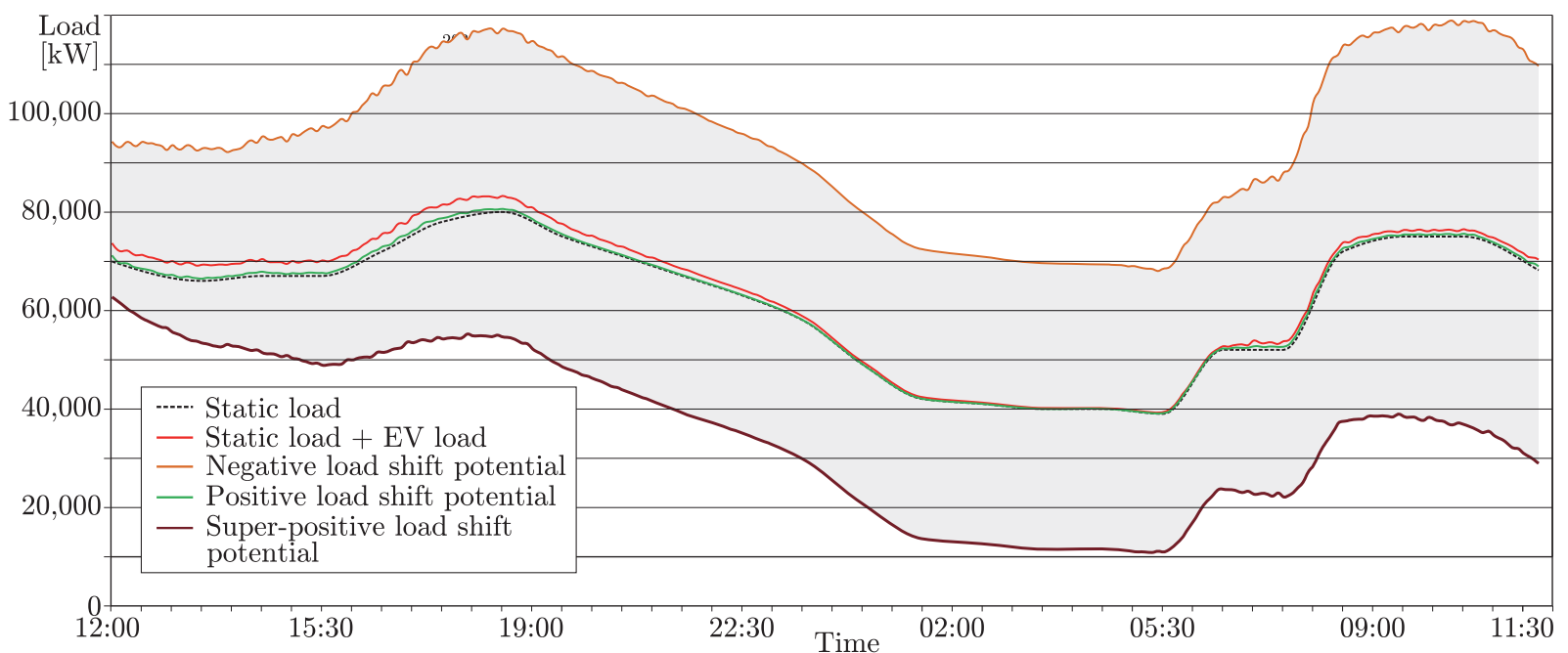

Figure 9. Resulting limits of load shift potentials (10,000 EV) in Scenario 2 (Year 2030).

\subsection{Scenario 2 (Year 2030)}

Again based on the NPE goals [24] and the German mobility study [25], a non-linear extrapolation leads to an estimated number of 6 million EV in Germany in the year 2030 (Figure 6). Due to the assumed increasing number of pure EV and the decreasing share of hybrid $\mathrm{EV}$ as well as the expected development of battery technology, the average battery capacity is significantly higher than in the year 2015 (cf. Table 1). Furthermore, rising acceptance for V2G measures is expected, which is why the share of participating vehicles is set to $50 \%$.

The results given in Table $\mathbf{3}$ differ significantly from those of Scenario 1. Due to the increased average battery capacity, the aggregated load shift potential increases at almost all points of the simulation. Consequently, the DSM has higher degrees of freedom in utilizing load shifting potentials. Especially the rise in the super-po- sitive load shift potential is remarkable. This may be explained by the higher share of vehicles participating in V2G measures.

To assess the results, a short example will be given below. The released positive secondary power reserve in Germany from October 24 to 30, 2011 reached a peak of approx. $2 \mathrm{GW}$. This means that the providers have to decrease their loads or to feed electricity into the grid. This form of power reserve can only be provided by vehicles which take part in V2G measures. In our nomenclature, the sum of positive and super-positive load shift potential serves to fulfill requests of positive reserve power. The results in Table 3 show that the peak demand in the week considered could have been met by EV at every time in the time horizon of the simulation. However, the duration of the request and the profitability for the customer [26] have a significant influence on its feasibility. 
Table 2. Selected scaled-up simulation results of Scenario 1 (2015).

\begin{tabular}{cccccc}
\hline & \multirow{2}{*}{ EV load } & \multicolumn{3}{c}{ Load shift potential [kW] } & \multirow{2}{*}{$\begin{array}{c}\text { Number } \\
\text { of EV }\end{array}$} \\
\cline { 3 - 5 } & & Negative & Positive & Super-p. & \\
\hline 1:00 pm & 30,529 & 70,724 & 26,422 & 21,363 & 33,729 \\
6:00 pm & 26,614 & 90,552 & 21,739 & 50,603 & 63,870 \\
3:00 am & 1903 & 61,127 & 1718 & 57,434 & 76,281 \\
8:00 am & 22,019 & 109,945 & 15,758 & 66,233 & 79,145 \\
Max. & 36,951 & 115,365 & 29,497 & 82,894 & 90,994 \\
Min. & 1112 & 59,445 & 987 & 12,907 & 26,075 \\
\hline
\end{tabular}

Table 3. Selected scaled-up simulation results of Scenario 2 (2030).

\begin{tabular}{cccccc}
\hline \multirow{2}{*}{ EV load } & \multicolumn{3}{c}{ Load shift potential [MW] } & \multirow{2}{*}{ Number of } \\
\cline { 3 - 5 } & & Negative & Positive & Super-p. & EV \\
\hline 1:00 pm & 2067 & 14,122 & 1665 & 6555 & $1.824 \mathrm{M}$ \\
6:00 pm & 2241 & 20,078 & 1749 & 14,713 & $3.445 \mathrm{M}$ \\
3:00 am & 150 & 17,675 & 143 & 17,055 & $4.098 \mathrm{M}$ \\
8:00 am & 1414 & 23,183 & 821 & 19,730 & $4.257 \mathrm{M}$ \\
Max. & 2347 & 25,596 & 1795 & 23,538 & $4.873 \mathrm{M}$ \\
Min. & 94 & 12,376 & 86 & 4330 & $1.408 \mathrm{M}$ \\
\hline
\end{tabular}

\subsection{Load Shifting}

As explained in 4, the simulation result highly depends on the temporal resolution. Choosing a larger time step gives information on the attainable load shift over a longer time period. Figure $\mathbf{1 0}$ shows the remaining average potential in percent in comparison to the 5-minute time step used for the examples above. The difference between 2015 and 2030 results from the higher participation in DSM measures, allowing higher short-term shifts. Figure 11 shows the result of a load shift of $+1500 \mathrm{~kW}$ from 23:00 to 0:00 (approx. 25\% of the $5 \mathrm{~min}$. potential at 23:00) for the 2015 scenario. Depending on the given tariff, the effects of a shift might be visible for several hours: The vehicles are forced to charge during 23:00 and 0:00, reduce their power or even discharge from 0:00 to 5:00, and fill up the battery between 5:00 and 6:00 because of a slightly cheaper tariff level (Figure 7).

Meanwhile, the negative potential is reduced to 2000 $\mathrm{kW}$ during the shift and considerably decreased afterwards. This information could be used for drafting riskbased DSM algorithms using load shift potentials as a measure of remaining flexibility.

\section{Outlook}

The knowledge and software developed in the course of this analysis is highly relevant for DSO and will be the basis for various further developments, from grid development planning to drafting business models.

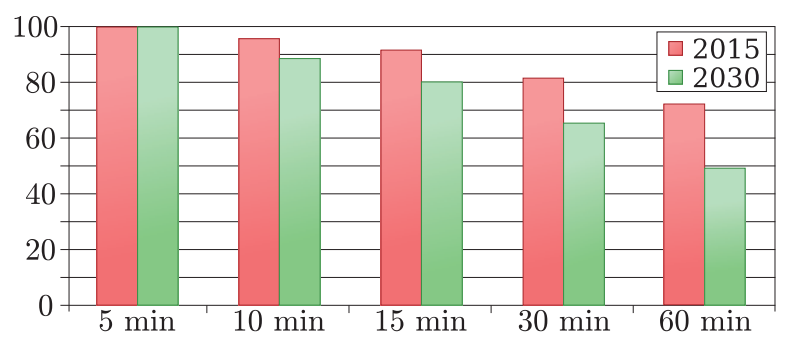

Figure 10. Remaining negative shift potential in percent for larger time steps.

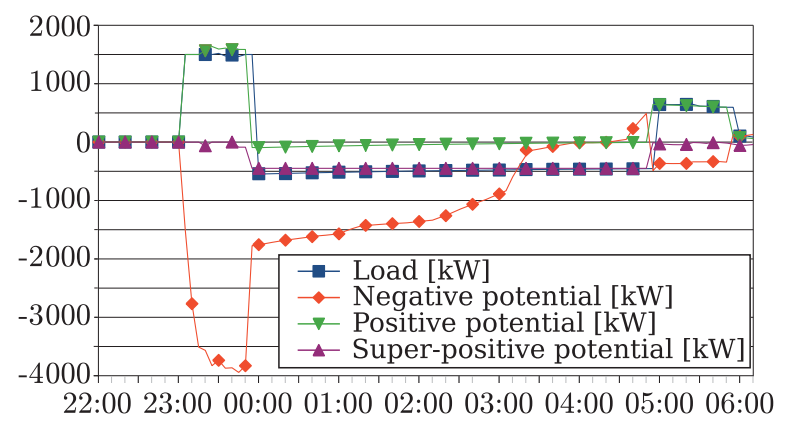

Figure 11. Resulting absolute change in load and potentials when performing a shift of $1500 \mathrm{~kW}$ from 23:00 to 0:00.

Real battery data or advanced battery models should be used in further studies to assess the real wear of the vehicle batteries. More reliable data for the future distribution of EV and their corresponding user patterns would also improve the results.

Stochastic influences should be included as well to simulate customer reactions to unforeseen events and the partly unreliable communication between the agents. Not only flexible and robust algorithms, but also communication protocols are needed for more realistic simulations.

Currently, no grid-imposed constraints are included. Due to fast charging stations at home, a shortage in the local grid might occur especially in the evening hours. Besides the lowest-voltage grid, also the low- and medium-voltage grid might be affected in some regions [26]. Furthermore, the time of charging has an impact on the specific $\mathrm{CO}_{2}$ emissions (and other emissions) during $\mathrm{EV}$ usage due to the time-dependent energy mix [27]. Both issues could be incorporated in future studies.

In addition, simulations of the actual power flow will have to be applied to include grid topology. This will be achieved by coupling the algorithms for optimal power flow computations presented in [28] with the existing software. An adaptive algorithm for optimal control of the distributed local grid as a function of the flexible demand of other users or even flexible or fluctuating electricity supply by combined heat and power units or photovoltaic systems might be an interesting extension of this project. The variety of proposed DSM algorithms (P2P, heuristic, deterministic) can be evaluated for dif- 
ferent scenarios in the future, with $\mathrm{CO}_{2}$ minimizing strategies being taken into account.

\section{Conclusions}

A bottom-up simulation algorithm is presented, which allows for determining the cumulative load curve and load shifting potentials of $\mathrm{EV}$ in the charging process in a generic energy grid. A direct algorithm was developed for this purpose that can be efficiently applied to all vehicles in parallel.

For a manual change of the grid load by requesting load shifts, a cost-optimal shift is performed by altering the original charging curve of some participating vehicles within their individual constraints, e.g. departure with desired state of charge of the battery. A mixed-integer linear program (MIP) has been developed that can be solved more efficiently than the original non-convex formulation.

Results of two scenarios based on mobility studies show that the load shifting potential of EV is significant and contribute to a necessary relaxation of the future grid. The maximum charging and discharging power and the average battery capacity are crucial to the overall load shift potential. However, the individual willingness to participate will depend on the profitability for the customer, which is why future projects should pay particular attention to this issue.

\section{Acknowledgements}

This work was supported by the Energy Solution Center (EnSoC), an association of major industrial corporations and research institutions in Germany. EnSoC focuses on projects using high-performance computing technology in application-oriented energy research. The authors would like to thank EnSoC for the continuous support and the professional project management.

We acknowledge support by Deutsche Forschungsgemeinschaft and Open Access Publishing Fund of Karlsruhe Institute of Technology.

\section{REFERENCES}

[1] C. Weiller, "Plug-In Hybrid Electric Vehicle Impacts on Hourly Electricity Demand in the United States," Energy Policy, Vol. 39, No. 6, 2011, pp. 3766-3778. doi:10.1016/j.enpol.2011.04.005

[2] T. Pollok, C. Matrose, T. Dederichs, A. Schnettler and E. Szczechowicz, "Classification and Comparison of Multi Agent Based Control Strategies for Electric Vehicles in Distribution Networks," Proceedings of 21st International Conference and Exhibition on Electricity Distribution (CIRED 2011), 6-9 June 2011, Paper No 0974.

[3] K. Clement-Nyns, E. Haesen and J. Driesen, “The Impact of Charging Plug-In Hybrid Electric Vehicles on a Resi- dential Distribution Grid,” IEEE Transactions on Power Systems, Vol. 25, No. 1, 2010, pp. 371-380. doi:10.1109/TPWRS.2009.2036481

[4] N. Hartmann and E. D. Özdemir, "Impact of Different Utilization Scenarios of Electric Vehicles on the German Grid in 2030,” Journal of Power Sources, Vol. 196, No. 4, 2010, pp. 2311-2318. doi:10.1016/j.jpowsour.2010.09.117

[5] H. Lund and W. Kempton, "Integration of Renewable Energy into the Transport and Electricity Sectors through V2G,” Energy Policy, Vol. 36, No. 9, 2010, pp. 35783587. doi:10.1016/j.enpol.2008.06.007

[6] D. Dallinger, D. Krampe and M. Wietschel, "Vehicleto-Grid Regulation Reserves Based on a Dynamic Simulation of Mobility Behavior," IEEE Transactions on Smart Grid, Vol. 2, No. 2, 2011, pp. 302-313. doi:10.1109/TSG.2011.2131692

[7] M. Agsten, S. Schlegel and D. Westermann, "On the Optimization of the Load of Electric Vehicles," Proceedings of the 18th IFAC World Congress, Milano, 28 August-2 September 2011, pp. 13673-13678.

[8] C. Ahn, C. T. Li and H. Peng, "Optimal Decentralized Charging Control Algorithm for Electrified Vehicles Connected to Smart Grid," Journal of Power Sources, Vol. 196, No. 23, 2011, pp. 10369-10379. doi:10.1016/j.jpowsour.2011.06.093

[9] O. Sundström and C. Binding, "Flexible Charging Optimization for Electric Vehicles Considering Distribution Grid Constraints,” IEEE Transactions on Smart Grid, Vol. 3, No. 1, 2012, pp. 26-37. doi:10.1109/TSG.2011.2168431

[10] P. Jochem, T. Kaschub, A. G. Paetz and W. Fichtner, "Integrating Electric Vehicles into the German Electricity Grid-An Interdisciplinary Analysis,” Proceedings of Electric Vehicle Symposium EVS26, Los Angeles, 6-9 May 2012, pp. 1272-1279.

[11] L. Göransson, S. Karlsson and F. Johnsson, "Plug-In Hybrid Electric Vehicles as a Mean to Reduce $\mathrm{CO}_{2}$ Emissions from Electricity Production," Proceedings to Electric Vehicle Symposium EVS24, Stavanger, 13-16 May 2009, pp. 2614-2624.

[12] S. Inage, "Modelling Load Shifting Using Electric Vehicles in a Smart Grid Environment," IEA Energy Papers, No. 2010/07, OECD Publishing, Paris, 2010. doi:10.1787/5km7v0bsmw7k-en

[13] M. G. Vaya and G. Andersson, "Locational Marginal Pricing Based Smart Charging of Plug-In Hybrid Vehicle Fleets," Proceedings of the Smart Energy Strategies Conference, Zurich, 21-23 September 2011.

[14] A. G. Paetz, T. Kaschub, M. Kopp, P. Jochem and W. Fichtner, "Monetäre Anreize zur Steuerung der Ladelast von Elektrofahrzeugen-Eine Modellgestützte Optimierung," Zeitschrift für Energiewirtschaft, Vol. 37, No. 1, 2013, pp. 1-12. doi:10.1007/s12398-012-0095-z

[15] S. Stein, E. Gerding, V. Robu and N. R. Jennings, “A Model-Based Online Mechanism with Pre-Commitment and Its Application to Electric Vehicle Charging," Proceedings of the International Conference on Autonomous 
Agents and Multiagent Systems AAMAS12, Valencia, 4-8 June 2012, pp. 669-676.

[16] S. Hutterer, M. Affenzeller and F. Auinger, "Evolutionary Optimization of Multi-Agent Control Strategies for Electric Vehicle Charging," Proceedings of the 14th International Conference on Genetic and Evolutionary Computation Conference Companion, Philadelphia, 7-11 July 2012, pp. 3-10. doi:10.1145/2330784.2330786

[17] J. Oyarzabal, J. Jimeno, J. Ruela, A. Engler and C. Hardt, “Agent Based Micro Grid Management System," Proceedings of the International Conference on Future Power Systems, Amsterdam, 18 November 2005, pp. 1-6.

[18] A.-G. Paetz, B. Becker, W. Fichtner and H. Schmeck, "Shifting Electricity Demand with Smart Home Technologies-An Experimental Study on User Acceptance," 30th USAEE/IAEE North American Conference Online Proceedings, Washington DC, 9-12 October 2011.

[19] R. Horst and H. Tuy, "Global Optimization: Deterministic Approaches,” Springer, Berlin Heidelberg, 2003.

[20] J. M. Zamora and I. E. Grossmann, “A Branch and Contract Algorithm for Problems with Concave Univariate, Bilinear and Linear Fractional Terms,” Journal of Global Optimization, Vol. 14, No. 3, 1999, pp. 217-249. doi:10.1023/A:1008312714792

[21] A. H. Land and A. G. Doig, "An Automatic Method of Solving Discrete Programming Problems,” Econometrica, Vol. 28, No. 3, 1960, pp. 497-520. doi:10.2307/1910129

[22] M. Berkelaar, K. Eikland and P. Notebaert, "lp_solve-A Mixed Integer Linear Programming (MILP) Solver,” Version 5.5.2.0, 2011. http://lpsolve.sourceforge.net/5.5

\section{Nomenclature}

\section{Load Curve}

$L_{V} \quad$ Continuous load curve of Vehicle V

$\bar{L}_{V} \quad$ Vectorized, piece-wise constant load curve

$\bar{Z}_{V}^{+} \quad$ Positive part of vectorized load curve

$\bar{Z}_{V}^{-} \quad$ Negative part of vectorized load curve

\section{Vehicle Properties}

$T_{V} \quad$ Time interval of connection

$E_{V}^{+}$Amount of energy until full charge

$E_{V}^{-} \quad$ Amount of energy dischargeable

$P_{V \text {,min }}^{+}$Minimum charge power

$P_{V, \text { max }}^{+}$Maximum charge power

$P_{V \text {,min }}^{-}$Minimum discharge power

$P_{V, \text { max }}^{-}$Maximum discharge power

$\bar{C}_{V}^{-}$Binary charge indicator

$\bar{F}_{V}^{-} \quad$ Binary feed indicator
[23] D. Vollmer, P. Horstmann and M. Kneifel, "Elektrische Standklimatisierung in Hybridfahrzeugen,” 2011. http://co2-testzentrum.de/download/symposium_06/Man Bosch. pdf

[24] National Electric Mobility Platform (Nationale Plattform Elektromobilität), "Zweiter Bericht der Nationalen Plattform Elektromobilität,” Gemeinsame Geschäftsstelle Elektromobilität der Bundesregierung, Berlin, 2011.

[25] Infas and DLR, “Mobilität in Deutschland 2008,” Institute for Applied Social Sciences GmbH (Infas), German Aerospace Center (DLR), on Behalf of the Federal Ministry of Transport, Building and Urban Development (BMVBS), 2010.

[26] P. Jochem, T. Kaschub and W. Fichtner, "How to Integrate Electric Vehicles in the Future Energy System?” In: M. Hülsmann and D. Fornahl, Eds., Evolutionary Paths towards the Mobility Patterns of the Future, Springer, Heidelberg, 2012.

[27] H. Heinrichs, A. Eßer-Frey, P. Jochem and W. Fichtner, "Zur Analyse der Langfristigen Entwicklung des Deutschen Kraftwerkparks-Zwischen Europäischem Energieverbund und dezentraler Erzeugung,” In: VDI-GET, Ed., Optimierung in der Energiewirtschaft, VDI Verlag, Düsseldorf, 2011, pp. 19-30.

[28] M. Schoenfelder, A. Eßer-Frey, M. Schick, W. Fichtner, V. Heuveline and T. Leibfried, "New Developments in Modeling Network Constraints in Techno-economic Energy System Expansion Planning Models,” Zeitschrift für Energiewirtschaft, Vol. 36, No. 1, 2012, pp. 27-35. doi:10.1007/s12398-011-0067-8

\section{Load Shift Potential}

$S_{V} \quad$ Continuous bounded load shift potential (LSP)

$\bar{S}_{V} \quad$ Piece-wise constant bounded LSP

$S_{V, \text { min }}^{+}$Lower boundary of LSP through charging

$S_{V, \text { max }}^{+}$Upper boundary of LSP through charging

$S_{V}^{-}$min Upper boundary of LSP through feeding

$S_{V, \text { max }}^{-}$Lower boundary of LSP through feeding

$R_{V}^{-} \quad$ Negative LSP

$R_{V}^{+} \quad$ Positive LSP

$R_{V}^{*} \quad$ Super-positive LSP

\section{System Properties}

$W \quad$ Tariff/weight function

$\bar{W}^{+}$Piece-wise constant charge tariff

$\bar{W}^{-}$Piece-wise constant feed tariff 